PACS 61.72.-y, 68.43.-h, 72.40.+w, 76.60.-k, 81.40.Rs

\title{
Magnetic field-stimulated change of photovoltage in solar silicon crystals
}

\author{
O.O. Korotchenkov, L.P. Steblenko, A.O. Podolyan, D.V. Kalinichenko, P.O. Tesel'ko, \\ V.M. Kravchenko, N.V. Tkach \\ Taras Shevchenko Kyiv National University, Physics Department, \\ 4, prosp. Akademika Glushkova, Kyiv, Ukraine
}

\begin{abstract}
The effect of static magnetic field $(B=0.17 \mathrm{~T})$ on composition of defects and lifetime of charge carriers in solar silicon crystals has been investigated. Studied in this work was the character of changes in electrical characteristic of solar silicon. These changes are dependent on the time elapsed after the magnetic treatment. The results have been discussed in terms of spin-dependent processes in the subsystem of structural defects.
\end{abstract}

Keywords: magnetic field, solar silicon crystal, surface photovoltage, structure relaxation, charged defects, adsorption.

Manuscript received 28.08.12; revised version received 19.11.12; accepted for publication 26.01.13; published online 28.02.13.

\section{Introduction}

In recent years, weak magnetic field-stimulated magnetosensitive phenomena in weak-magnetic crystals are of increased interest. This kind of physical phenomena is taken into account by the models involving spin-dependent intercombination transitions in pairs of non-equilibrium paramagnetic defects [1-4]. The probability of decay of a structural complex containing at least two radicals each of which has a spin angular momentum depending on mutual orientation of the spins. Magnetic field (MF) is believed to have an influence on the probability of initiation of favourable orientation and stimulates singlet-triplet transitions. Spin conversion, in turn, shifts the chemical solid-state reaction towards decay.

By now, a large amount of experimental data has been accumulated, and a number of interesting effects has been detected $[5,6]$ in systematic investigations of the effect of weak magnetic fields $(B \leq 1 \mathrm{~T})$ on magnetically disorded (weak-magnetic) materials. Taking into account the fact that silicon is the basic structural material of microelectronics, the portion of the mentioned studies on silicon crystals is unwarrantably small.

Especially noticeable gaps exist in the knowledge of magnetostimulated changes in the electrophysical characteristics of silicon. The respective studies have not been carried out on solar silicon (SS) crystals at all. Meanwhile, that kind of studies is urgent and worthwhile, since microelectronic devices, manufactured on the SS basis, most often operate under extreme conditions, including the action of magnetic fields. Therefore, the operation of SS-based devices must be predictable and reliable.

It should be noted that investigation of electrophysical characteristics of SS such as photovoltage makes it possible to obtain important information on the electron subsystem of SS crystals. That kind of studies also provide particular information on interaction of charge carriers (photocarriers) generated by absorption of electromagnetic radiation (photons) with inhomogeneities caused by structural peculiarities in SS crystals. 
A lack of studies concerning the action of magnetic field on photovoltage in SS crystals and practical necessity of such investigations make the present work quite appropriate. The aim was to reveal changes in the photovoltage magnitude and decay behaviour caused by the action of a weak constant magnetic field.

\section{Experimental}

Boron-doped Czochralski-grown solar Si crystals with the resistivity $\rho=5 \mathrm{Ohm} \cdot \mathrm{cm}$ were studied. Magnetic treatment (MT) of SS samples consisted in exposing them to constant magnetic field with an induction of $B=0.17 \mathrm{~T}$ during certain period of time lasting from $\mathrm{t}_{\mathrm{MT}}=5$ to 30 days. Photovoltage measurements were carried out by the contactless capacitance method. The principles of this method are described in [7]. Charge carriers were generated by light pulses from a LED with emission spectrum peak at $650 \mathrm{~nm}$ and pulse duration of a few microseconds. All this provided the surface generation of charge carriers.

\section{Results and discussion}

The measured photovoltage decay kinetics show two relaxation components: fast and slow ones (Fig. 1). Using the method of approximation, we distinguished fast $\left(\tau_{1}\right)$ and slow $\left(\tau_{2}\right)$ components of the charge carrier lifetime in both the reference SS samples and the magnetic-field-treated ones.

Fig. 2 illustrates a change in charge carrier lifetime with duration of MT. One can see that magnetic treatment for $t_{\mathrm{MT}}=5$ days results in a decrease in both fast $\left(\tau_{1}\right)$ and slow $\left(\tau_{2}\right)$ lifetime components. Both components fall approximately 3 times. Further prolongation of MT duration $\left(t_{\mathrm{MT}}=30\right.$ days $)$ does not affect $\tau_{1}$ and $\tau_{2}$.

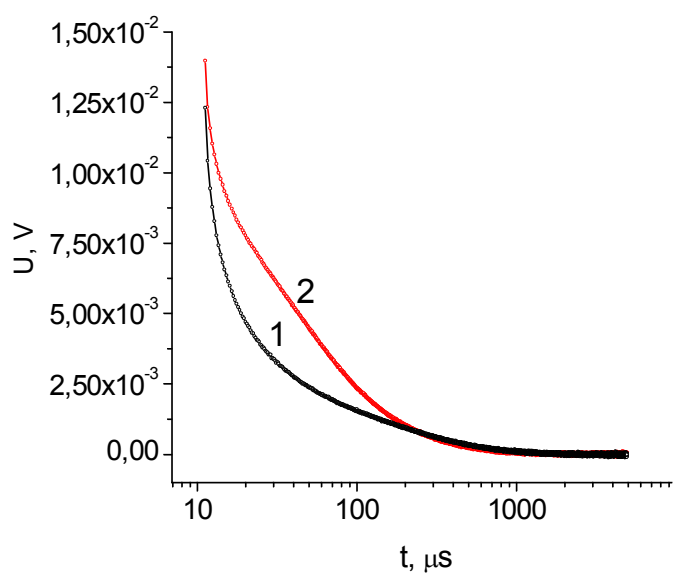

Fig. 1. Photovoltage decay kinetics in SS samples: the reference sample ( 1$)$ and the sample exposed to magnetic field for 21 days (2).
In this work, we studied not only the effect of lifetime change as a result of MT with varied duration, which was detected directly after MT, but we also examined the lifetime constancy. It was found that after MT termination the slow photovoltage decay time constant $\tau_{2}$ remains unchanged, while the fast one $\tau_{1}$ decreases slowly with time (Fig. 3). On the whole, the time constant $\tau_{1}$ fell approximately 5 times for the first 30 days and then remained almost constant for 230 days.

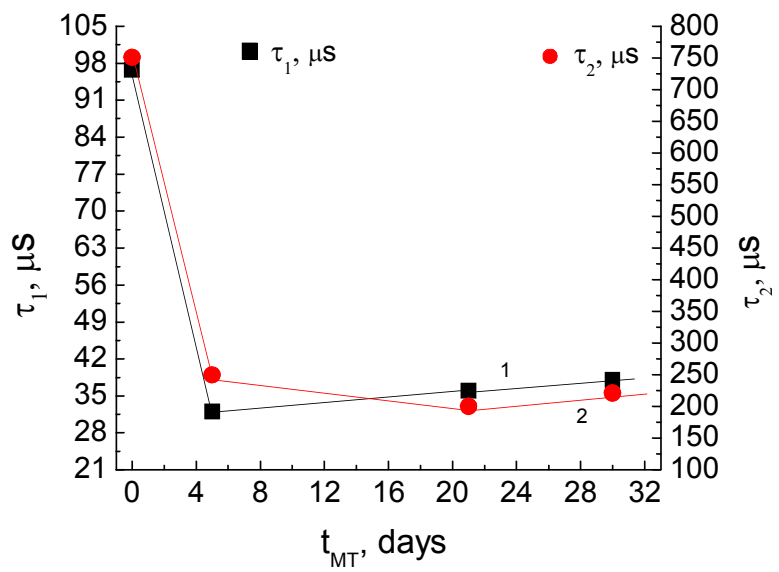

Fig. 2. Dependence of fast (1) and slow (2) photovoltage decay time constants on duration of magnetic treatment of SS samples.
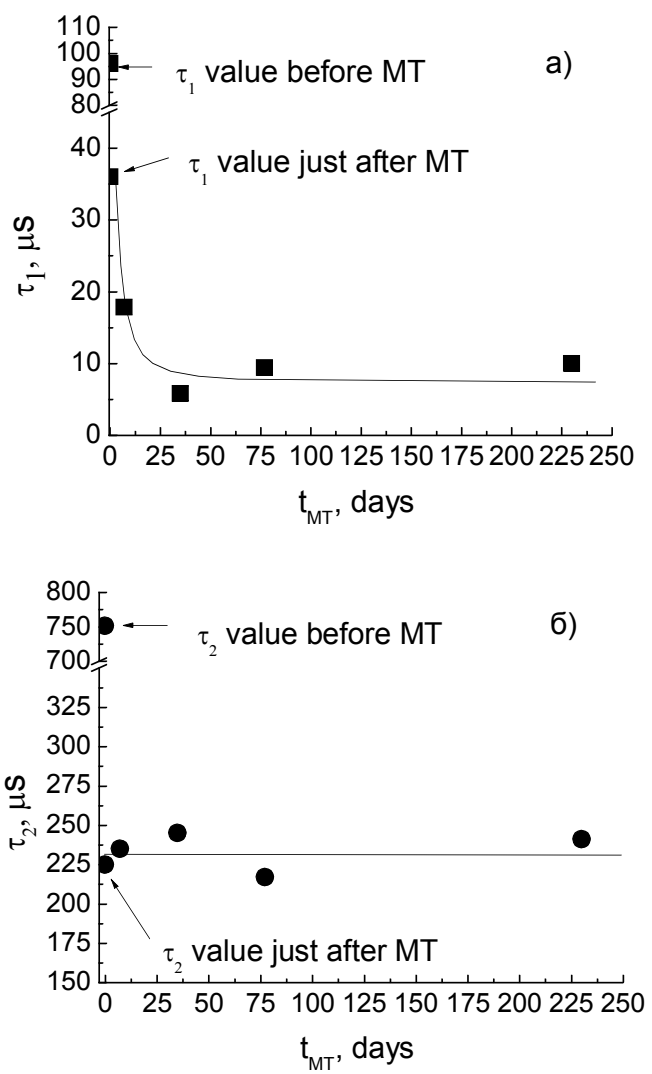

Fig. 3. Fast $(a)$ and slow $(b)$ photovoltage decay time constants vs time that elapsed after MT termination. 
Explanation of the results obtained may be based on the following considerations. A decrease in the time constant after 5-day-long exposition to magnetic field can be attributed to magnetic field-stimulated breakage of chemical bonds in structural nanoclusters, first of all, the bonds in oxide precipitates $\left(\mathrm{SiO}_{\mathrm{x}}\right)$ and hydride groups $(\mathrm{SiH})$, which predominate in silicon. Some saturation observed on the dependence of $\tau$ on $t_{\mathrm{MT}}$ (Fig. 2) may be due to the fact that after 5 days the process of spin-dependent decay of structural nanoclusters in silicon had finished and further prolongation of MT duration had no effect. Point defect complex decay itself results in appearance of additional defects in the form of dangling bonds. Among newly formed defects, there are also electrically active defects which act as recombination centers. In our opinion, in addition to electrically active dangling bonds, the defects of that kind may be defects in the form of non-bridge oxygen. It is known from literature that in the system "silicon - silicon oxide" (it is the system that we deal with in our experiments) the $\mathrm{Si}-\mathrm{SiO}_{2}$ interface contains certain quantity of such defects as non-bridge silicon (oxygen radicals that is not saturated by silicon) [8].

Non-bridge oxygen is a charged defect [8], it can act as a recombination center and decrease the charge carrier lifetime. Moreover, as shown in our previous work [9], negative electric charge formed on the $\mathrm{Si}-\mathrm{SiO}_{2}$ interface under action of magnetic field stimulates the process of surface gettering the positive ions of alkali metals $\mathrm{K}^{+}, \mathrm{Na}^{+}, \mathrm{Ca}^{+}$and $\mathrm{Al}^{+}$which are present in $\mathrm{Si}$ bulk. These ions can also act as recombination centers and decrease the charge carrier lifetime as a result of 5day-long MT.

Table 1. Distribution of carbon and oxygen impurities as well as the host element silicon in the initial SS sample.

\begin{tabular}{|c|c|c|c|}
\hline Spectrum No. & C, wt.\% & O, wt.\% & Si, wt.\% \\
\hline 1 & 1.43 & 0.19 & 98.57 \\
\hline 2 & 1.79 & 0.31 & 98.21 \\
\hline 3 & 1.59 & 0.33 & 98.41 \\
\hline 4 & 5.82 & 0.03 & 93.72 \\
\hline 5 & 1.65 & & 98.35 \\
\hline Mean & 2.45 & 0.21 & 97.45 \\
\hline Max. & 5.82 & 0.33 & 98.57 \\
\hline Min. & 1.43 & 0.03 & 93.72 \\
\hline
\end{tabular}

Table 2. Distribution of carbon and oxygen impurities as well as the host element silicon in the SS sample subjected to MT.

\begin{tabular}{|c|c|c|c|}
\hline Spectrum No. & C, wt.\% & O, wt.\% & Si, wt.\% \\
\hline 1 & 10.32 & 0.50 & 89.18 \\
\hline 2 & 11.09 & 0.47 & 88.44 \\
\hline 3 & 5.00 & 0.13 & 94.87 \\
\hline 4 & 4.51 & 0.21 & 95.28 \\
\hline Mean & 7.73 & 0.33 & 91.94 \\
\hline Max. & 11.09 & 0.50 & 95.28 \\
\hline Min. & 4.51 & 0.13 & 88.44 \\
\hline
\end{tabular}

Our studies of impurity composition of the SS samples by using X-ray spectroscopy have shown that distribution of carbon and oxygen impurities as well as the host element silicon in the near-surface layers of the SS crystals is non-uniform (Table 1).

The MT somewhat increases the degree of nonuniformity in the distribution of the above-mentioned impurities (Table 2).

As already noted, MT-modified $\tau_{2}$ component remains constant for a long time (230 days). Taking into account the fact that barrier-layer photovoltage (it is the type of photovoltage that we deal with) is generated in chemically heterogeneous and nonuniformly doped semiconductors, one can suppose that the effect of long-lasting invariability of the $\tau_{2}$ component of photovoltage decay in SS after MT may be due to existence of constant (invariable with time) structural inhomogeneity in the bulk part of the SS sample.

At the same time, the effect of long-lasting decrease in the fast component $\tau_{1}$ after MT may be attributed to not only structural inhomogeneity in the bulk part of the SS but also structural inhomogeneity of the surface. In our opinion, it is the defect and impurity state of the surface that changes as time interval between MT termination and measurement of photovoltage decay kinetics rises. Let us dwell on this point in more detail. It is known from literature $[9,10]$ that, in addition to the process of chemical bond breakage in point defect complexes, magnetic field also stimulates the processes of adsorption and gettering the impurities on MTactivated surface.

An adsorbed particle can be considered as a donor or acceptor impurity. Adsorbed particles may be charged or neutral and may exchange charge carriers with the crystal. So, the process of adsorption can generate new energy levels at the semiconductor interface. It is not inconceivable that, under conditions of our experiment on the MT-activated surface, adsorbed are hydroxyl groups and oxygen from the ambient atmosphere. These adsorbed centers, number of which rises with time, generate additional surface electron states on the $\mathrm{Si}$ surface and, consequently, cause a decrease in the charge carrier lifetime during some 30 days after MT termination.

An MT-induced increase in the degree of structural inhomogeneity in SS led not only to changes in the electro-physical characteristics (photovoltage decay kinetics) but also to changes in internal stresses which result in sample bending, i.e. to alteration of the radius of curvature of atomic planes. Complementary studies of sample deformation were carried out by X-ray diffractometric measurements of the curvature of atomic planes. It was found that MT decreases the radius of curvature. The latter may be caused by MT-stimulated process of structural relaxation, which is the reason of a decrease in internal microstresses. 


\section{References}

1. Ya.B. Zeldovich, A.L. Buchachenko, E.L. Frankevich, Magnetic-spin effects in chemistry and molecular physics // Uspekhi Fiz. Nauk, 155(1), p. 3-45 (1988), in Russian.

2. M.I. Molotskiy, Possible mechanism of the magnetoplastic effect // Fizika Tverd. Tela, 33(10), p. 3112 (1991), in Russian.

3. A.L. Buchachenko, Magnetoplasticity of diamagnetic crystals in microwave fields // Zhurnal Eksperiment. Teor. Fiziki, 132(3), p. 673-679 (2007), in Russian.

4. A.L. Buchachenko, Physical kinetics of magnetoplasticity for diamagnetic crystals // Zhurnal Eksperiment. Teor. Fiziki, 132(4), p. $827-$ 830 (2007), in Russian.

5. R.B. Morgunov, Spin micromechanics in the physics of plasticity // Uspekhi Fiz. Nauk, 174(2), p. 131-153 (2004), in Russian.
6. Yu.I. Golovin, Magnetoplasticity of solids (Review) // Fizika Tverd. Tela, 46(5), p. 769-803 (2004), in Russian.

7. L. Kronik and Y. Shapira, Surface photovoltage techniques: Theory, experiment, and applications // Surf. Sci. Repts. 37(1-5), p. 1-206 (1999).

8. V.S. Vavilov, V.F. Kiselev, B.N. Mukashev, Defects in Silicon and on its Surface. Nauka Publ., Moscow, 1990 (in Russian).

9. V.A. Makara, M.A. Vasilyev, L.P. Steblenko et al. Magnetic-field-induced changes in the impurity state and microhardness of silicon crystals // Fizika Tekhnika Poluprovodnikov, 42(9), p. 1061-1064 (2008), in Russian.

10. M.N. Levin, A.V. Tatarintsev, O.A. Kostsov, A.M. Kostsov, Semiconductor surface activation by the action of pulsed magnetic field // Zhurnal Tekhnich. Fiziki, 73(10), p. 85-87 (2003), in Russian. 